\title{
Density wave patterns for fermionic dipolar molecules on a square optical lattice: Mean-field-theory analysis
}

\author{
K. Mikelsons and J. K. Freericks \\ Department of Physics, Georgetown University, Washington, DC, 20057 USA
}

(Dated: November 3, 2018)

\begin{abstract}
We model a system of ultracold fermionic dipolar molecules on a two-dimensional square lattice. Assuming that the molecules are in their nondegenerate hyperfine ground state, and that the dipole moment is polarized perpendicular to the plane (as in the recent experiments on ${ }^{40} \mathrm{~K}-{ }^{87} \mathrm{Rb}$ molecules), we approximate these molecules as spinless fermions with long range repulsive dipolar interactions. We use mean field theory to obtain the restricted phase diagram as a function of the filling, the strength of interaction and the temperature. We find a number of ordered density wave phases in the system, as well as phase separation between these phases. A Monte Carlo analysis shows that the higher-period phases are usually suppressed in the exact solution.
\end{abstract}

PACS numbers: 71.10.Fd,71.10.Hf,74.72.-h

\section{INTRODUCTION}

Recent experimental work [1] in ultracold molecule physics has formed fermionic dipolar molecules of ${ }^{40} \mathrm{~K}$ and ${ }^{87} \mathrm{Rb}$ in their rovibrational and hyperfine ground state. Quantum degenerate dipolar systems have long been sought after because their long-range interactions makes them interesting for quantum computing applications and for understanding ordered phases that can arise from long range effects. These systems are both complicated, but tunable, because the long range, anisotropic dipolar interaction can be manipulated with an external electric field [2], and should give rise to rich physics with variety of different ordered phases of matter [3].

The dipolar interaction is attractive if the dipole moments are aligned head to tail, and this can lead to undesirable recombination effects if the molecules have an exothermic reaction possible, as with ${ }^{40} \mathrm{~K}-{ }^{87} \mathrm{Rb}$ molecules [4]. This can be mitigated by confining molecules to a narrow two-dimensional layer, and setting a strong electric field perpendicular to this layer, so that the dipole moments of molecules are aligned with the external field and always align head-to-head and tail-to-tail. In this case, the inter-molecule interactions are always repulsive. Note that we assume we can create a single isolated plane of dipoles, so there is no possibility to form superfluid pairing between planes, where the interactions are attractive again [5].

We assume the dipolar molecules are loaded onto a single square optical lattice with the molecules fully polarized perpendicular to the plane. Since the ${ }^{40} \mathrm{~K}-{ }^{87} \mathrm{Rb}$ molecules can be prepared in their lowest hyperfine nuclear spin state, the internal spin degree of freedom is effectively frozen out, and the molecules can be described as spinless fermions.

Previous studies of spinless fermion models [6 8 ] mostly focused on short range interactions and used the simplification of going to the limit of large spatial dimensions. These works show a rich phase diagram with charge-density-wave ordering and phase separation. There also has been some work in two dimensions focused on stripe physics [9].

More recent work, carried out in the context of ultracold dipolar molecules [3, 10 12, focused primarily on finding new exotic phases (liquid crystal, smectic, or nematic), often achieved by tuning the direction of the external field with respect to the plane of molecules, but has not elucidated the details of the full phase diagram for the density-wave phases.

The paper is organized as follows: First, in Sec. II we introduce the model and describe the possible densitywave orderings. Sec. III provides the details of the meanfield-theory formalism and the numerical calculations. The results are presented in Sec. IV] and they are further discussed in Sec. $\nabla$, which are followed by the conclusions in Sec.VI.

\section{MODEL}

We describe the rovibrational ground-state dipolar molecules in their lowest hyperfine state with the following Hamiltonian:

$$
H=H_{k}+H_{p}=-t \sum_{\langle i j\rangle}\left(c_{i}^{\dagger} c_{j}+\text { h.c. }\right)+\sum_{i \neq j} U_{i j} n_{i} n_{j}
$$

where $c_{i}^{\dagger}\left(c_{i}\right)$ is the fermion creation (annihilation) operator for a fermionic dipolar molecule at site $i, n_{i}=c_{i}^{\dagger} c_{i}$ is the number operator, $t$ is the hopping amplitude between the adjacent sites, and $U_{i j}=U /\left|\vec{r}_{i}-\vec{r}_{j}\right|^{3}$ is the long-range dipole interaction, with $\vec{r}_{i}$ the position vector for the site $i$. The lattice spacing is taken to be equal to one. With this model, we have assumed that the hopping occurs only between nearest-neighbor sites, and that the molecules are always in the lowest level of the periodic optical lattice potential (this second assumption is not always true when the interaction between the molecules is too large). We have also assumed that the form for the dipole interaction is valid even when the distance between molecules is only one lattice spacing. 
Due to the bipartite nature of the square lattice, the Hamiltonian can be rewritten in a particle-hole symmetric form and one can restrict to fillings that satisfy $f=\langle n\rangle \leq 0.5$.

Since there is no spin degree of freedom in this model, it can only support molecule density wave spatial order. Such order is described by its unit cell - a parallelogram spanned by the two fundamental translation vectors for a given density wave order. The area of the unit cell $\left(N_{c}\right)$ gives the number of sites with independent average molecule density which is modulated periodically as the unit cell is tiled across the lattice. The number of the independent order parameters for this density wave is equal to $N_{c}-1$ (the average density, or filling, is not counted as an order parameter, and is fixed in the calculation). Since there is only a finite number of non-equivalent unit cells of a given area on a square lattice, there is only a finite number of possible density wave orders. We have considered all such orders that have up to four different order parameters $\left(N_{c} \leq 5\right)$ and hence this is a restricted phase diagram restricted to low-period phases only. The corresponding fundamental translation vectors and the unit cells for the twelve candidate phases are shown in Fig. [1]

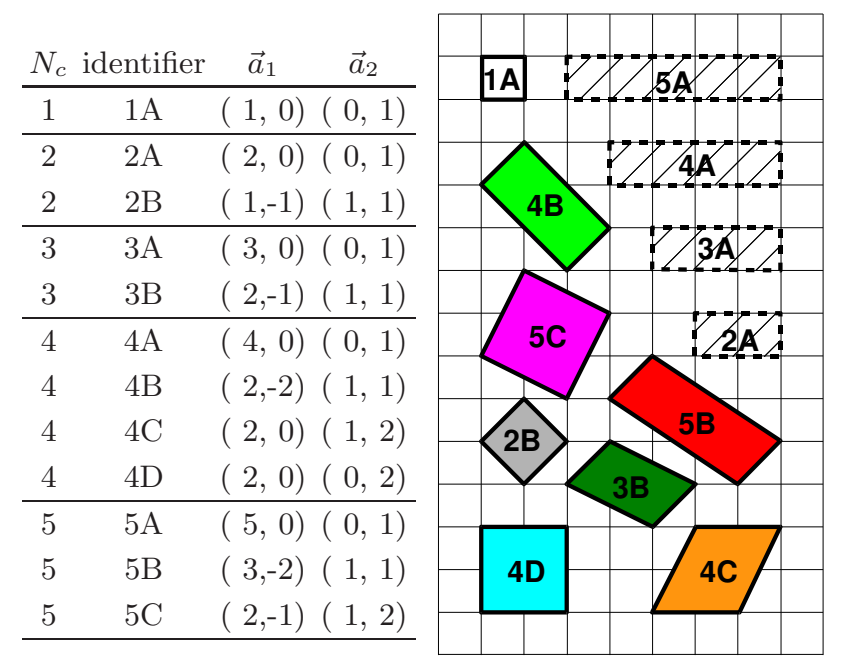

FIG. 1: Left: Fundamental translation vectors $\left(\vec{a}_{1}, \vec{a}_{2}\right)$ for possible density wave phases considered in this work, grouped by the area of the unit cell. Unit cell 1A describes a homogeneous system without order, while $2 \mathrm{~B}$ corresponds to checkerboard order. Right: Unit cells for the density wave phases. Only density wave orders corresponding to unit cells with the solid outline were found to be stabilized in this study.

\section{METHODOLOGY}

We solve the model using mean field theory (MFT). This can be justified, since the the interaction is long range and consequently each site is effectively coupled to any other site, thereby increasing the effective dimen- sion of the mean field and decreasing the role of fluctuations (nevertheless, this is only an approximate solution). In fact, due to the absence of a local interaction, the MFT is equivalent to the dynamical mean-field theory (DMFT) approach, which becomes exact in the infinite-dimensional limit. The absence of a spin degree of freedom also implies that the model is in the Ising universality class, with a finite transition temperature in 2D. Thus, while MFT is expected to overestimate the transition temperature, it is also expected to give the qualitatively correct phase diagram. Within MFT, the interaction part of Hamiltonian is approximated as:

$$
n_{i} n_{j} \approx n_{i}\left\langle n_{j}\right\rangle+\left\langle n_{i}\right\rangle n_{j}-\left\langle n_{i}\right\rangle\left\langle n_{j}\right\rangle,
$$

which means that the correlations of the density fluctuations are neglected. This corresponds to the first order (Hartree-Fock) self-consistent perturbation theory result and is expected to be accurate for small $U / t$.

In the MFT approximation, the order parameter $\left\langle n_{i}\right\rangle$ is a fixed parameter in Hamiltonian, and acts as a site dependent potential. The resultant MFT Hamiltonian is quadratic in the $\left(c, c^{\dagger}\right)$ operators and can be easily diagonalized. We generically work on a lattice with a large, but finite, number of lattice sites $N$ and periodic boundary conditions. Further simplification comes from exploiting translational invariance, which means that the Hamiltonian is block-diagonal in reciprocal space. The size of these blocks is $N_{c}$, and it grows with the number of the order parameters while the number of blocks, $N / N_{c}$, grows with the number of discretization points in reciprocal space, $N$, which is the same as the number of real-space lattice sites included in the calculation. The Hamiltonian in reciprocal space becomes:

$$
H=\sum_{\tilde{k}}^{N / N_{c}} \sum_{i j}^{N_{c}} c_{K_{i}+\tilde{k}}^{\dagger}\left[\left(\epsilon\left(\tilde{k}+K_{i}\right)-\mu\right) \delta_{i j}+V_{i j}\right] c_{K_{j}+\tilde{k}},
$$

where $\epsilon(k)=-2 t\left(\cos k_{x}+\cos k_{y}\right)$ is the tight-binding dispersion, $\mu$ is the chemical potential, $K_{i}$ are the reciprocal lattice points corresponding to the real-space basis vectors of a given order (see Fig. 1), and $\tilde{k}$ denotes the discrete summation points in the corresponding first Brillouin zone (FBZ) (see Fig. 21). The interaction part $V_{i j}=U\left(K_{i}-K_{j}\right)\left\langle m\left(K_{i}-K_{j}\right)\right\rangle$ where $m\left(K_{i}\right)$ is the Fourier transform of the real space density (order parameter): $m\left(K_{i}\right)=\sum_{j=1}^{N_{c}} e^{i K_{i} r_{j}} n_{j}$, and $U\left(K_{i}\right)$ is:

$$
U\left(K_{i}\right)=U \sum_{\text {all } j \neq 0} \frac{e^{i K_{i} r_{j}}}{\left|r_{j}\right|^{3}} .
$$

Since the the dipole interaction decays sufficiently fast with increasing distance, the sum can be carried out for an infinite range of distance, giving more precise values for the effective interaction in $k$-space. As seen from Eq. 3, $\tilde{k}$ is a good quantum number, while the spatially modulated mean field in the interaction term $V_{i j}$ causes 
molecule scattering among the $N_{c} K$-points. The calculation starts with a random guess for the order parameter $\left\langle n_{i}\right\rangle\left(i=1, \ldots, N_{c}-1\right)$, which is put into the Hamiltonian (Eq. [3), and the full set of eigenvalues $\left(\epsilon_{\tilde{k}, \alpha}\right)$ and the corresponding eigenvectors $\left[\psi_{\tilde{k} \alpha}\left(K_{i}\right)\right]$ are obtained by solving the eigenvalue equation (for all $\tilde{k}$ and $\alpha=1 \ldots N_{c}$ ):

$$
\sum_{j=1}^{N_{c}}\left[\left(\epsilon\left(\tilde{k}+K_{i}\right)-\mu\right) \delta_{i j}+V_{i j}\right] \psi_{\tilde{k} \alpha}\left(K_{j}\right)=\epsilon_{\tilde{k}, \alpha} \psi_{\tilde{k} \alpha}\left(K_{i}\right) .
$$

For simplicity, we denote $\epsilon_{i}=\epsilon_{\tilde{k} \alpha}$ and $\psi_{i j}=\psi_{\tilde{k} \alpha}\left(K_{j}\right)$. The energy per site and the filling fraction are next calculated via

$$
E=\frac{1}{N} \sum_{i=1}^{N} \epsilon_{i} n_{\epsilon_{i}} \text { and } f=\frac{1}{N} \sum_{i=1}^{N} n_{\epsilon_{i}},
$$

respectively, with $n_{\epsilon_{i}}=1 /\left(1+e^{\left(\epsilon_{i}-\mu\right) / T}\right)$ the Fermi-Dirac distribution and $T$ being the temperature. The meanfield entropy per site is

$$
S=-\frac{1}{N} \sum_{i=1}^{N}\left[n_{\epsilon_{i}} \ln \left(n_{\epsilon_{i}}\right)+\left(1-n_{\epsilon_{i}}\right) \ln \left(1-n_{\epsilon_{i}}\right)\right],
$$

and the free energy satisfies $F=E-T S$. The momentum dependent density is:

$$
\left\langle n_{\tilde{k}+K_{l}}\right\rangle=\left\langle c_{\tilde{k}+K_{l}}^{\dagger} c_{\tilde{k}+K_{l}}\right\rangle=\frac{1}{N_{c}} \sum_{i=1}^{N_{c}} n_{\epsilon_{i}} \psi_{i l} \psi_{i l}^{*} .
$$

Calculating the expectation value of the average real space density then yields the new values of the order parameter:

$$
\left\langle m\left(K_{j}\right)\right\rangle=-\frac{1}{N} \sum_{i=1}^{N} \sum_{l, m=1}^{N_{c}} n_{\epsilon_{i}} \psi_{i l} \psi_{i m}^{*} \delta\left(K_{l}-K_{m}-K_{j}\right),
$$

which can be used to calculate the structure factor $S(Q)=|m(Q)|^{2}$. These updated order parameter values are inserted back into the Hamiltonian in Eq. (3), and the iterative process is repeated until the order parameter has converged to a fixed point. The chemical potential needs to be adjusted after every MFT iteration, so as to maintain constant filling.

Solving the MFT equations represents finding a global minimum for the free energy functional in a multidimensional space. Since there can be multiple distinct stable solutions (corresponding to local minima in the free energy), the calculation needs to be repeated for several random initial choices of the order parameter, and only the solution corresponding to global free energy minimum should be taken. More starting points are necessary for higher order phases, since the parameter space of solutions is larger. For phases with only one order parameter (2B), the MFT solution can be found by a successive bisection method. Overall, we found that with 20 to 50 initial random order parameters, in more than half of the cases, the calculation converged to the lowest free energy minimum. The number of required integration points $N$ depends on the value of $U / t$ : for $U / t \rightarrow \infty$ taking $N=N_{c}$ is sufficient, while for small $U / t$ finer discretization is needed to account for the $k$-dependence of dispersion $\epsilon(k)$. For a given $U / t$, we started with a smaller number of integration points $N$, and increased it by a factor of four, until further increase did not produce a significant change in the phase diagram.

The search for the MFT solutions is repeated for all possible density-wave orders (as described above), and only the solution with the lowest free energy is ultimately taken.

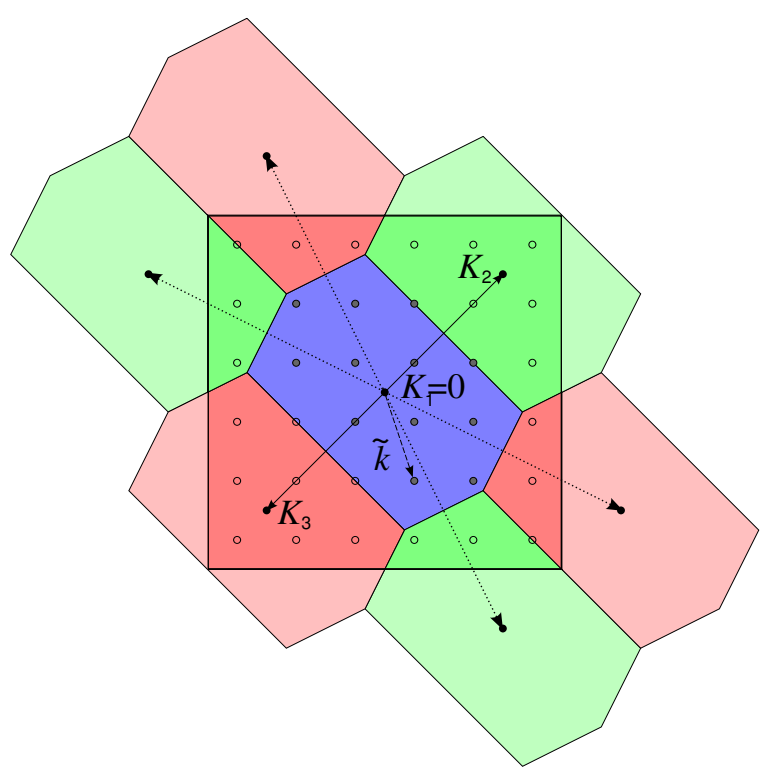

FIG. 2: (color online) Reciprocal space for the 3B order. The FBZ is shown in blue in the center of square. The black circles denote $K$ points $\left(N_{c}=3\right)$, associated with the density wave order, while the gray circles are the discretization $\tilde{k}$ points used in the calculation. The open circles denote other possible values of $K+\tilde{k}$ within reciprocal space. The dotted arrows denote other reciprocal space vectors for $3 \mathrm{~B}$ order used to construct the FBZ.

A simplification occurs in the limit $U / t \rightarrow \infty$. In this case, the kinetic energy term in the Hamiltonian in Eq. (11) is negligible, and the MFT Hamiltonian becomes:

$$
\begin{aligned}
H & =\sum_{i \neq j} U_{i j}\left[n_{i}\left\langle n_{j}\right\rangle+\left\langle n_{i}\right\rangle n_{j}-\left\langle n_{i}\right\rangle\left\langle n_{j}\right\rangle\right] \\
& =\sum_{i} W_{i} n_{i}+\text { const }
\end{aligned}
$$

with

$$
W_{i}=2 \sum_{j \neq i} U_{i j}\left\langle n_{j}\right\rangle
$$


The solution yields readily:

$$
\left\langle n_{i}\right\rangle=\left(1+e^{\left(W_{i}-\mu\right) / T}\right)^{-1} .
$$

Equations (12) and (13) need to be solved selfconsistently to yield the MFT result.

To account for the possibility of phase separation, we need to calculate the free energy for fixed $U / t$ and $T / t$ for full range of filling $f$ and perform a Maxwell construction. A clear indicator of phase separation is that the chemical potential dependence on the filling becomes multivalued (since $\mu=\partial F / \partial f$ ), as will be shown in the following Section.

\section{RESULTS}

We present results for the phase diagrams, obtained both with and without phase separation considered. To illustrate the procedure for detecting phase separation, we show results for the chemical potential at $T=0$ and $U / t=4$ in Fig. 3. The MFT solution is particle-hole symmetric, so that $\mu(f)+\mu(1-f)=2 \mu(0.5)$. However, it shows unphysical behavior at some regions where the chemical potential decreases with increasing filling. The physical solution avoids such regions by allowing for phase separation, which consists of mixtures of two different phases with the same chemical potential, at the same temperature and interaction $U / t$. As seen in Fig. 3. phase separation occurs at fillings slightly above or below the commensurate values, and is accompanied by a gap in the density of states at the commensurate values of filling.

The phase diagram for $U / t=\infty$ is shown in Fig. 4. In this case, the kinetic-energy part of the Hamiltonian is suppressed $(t=0)$, and the order at zero temperature extends to all values of the filling. The dominant order is the checkerboard pattern (2B), commensurate at half filling. At low temperature, higher-period orders emerge around the corresponding commensurate fillings. Yet low-period orders are more stable against thermal fluctuations, as indicated by their persistence up to higher temperatures. The transition between the high temperature homogeneous $(1 \mathrm{~A})$ to checkerboard phase $(2 \mathrm{~B})$ is continuous, as is the transition between the $2 \mathrm{~B}$ and $4 \mathrm{D}$ phases. The remaining phase transitions, which occur between higher-period phases at lower temperatures, are discontinuous and can involve phase separation.

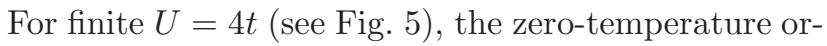
der is suppressed for small values of filling, yet the phase diagram around half-filling is relatively unchanged, as compared to $U / t=\infty$. Again, the checkerboard phase is dominant at higher temperatures. Higher-period phases (3B, 4C and 5B), although still confined to low temperatures, appear to be more protracted in the range of filling. The phase separation between these phases is also more prominent, but not qualitatively different than in the $U / t=\infty$ case. An interesting feature is the reentrant

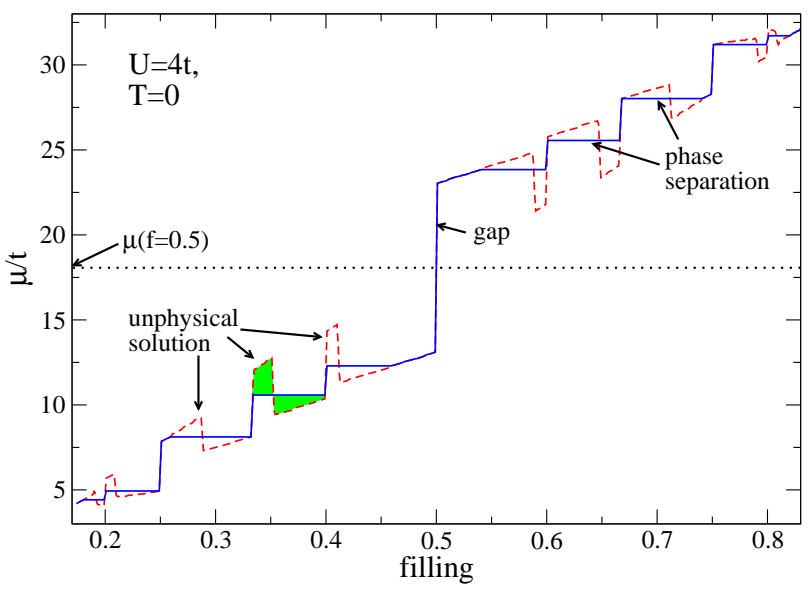

FIG. 3: (color online) Chemical potential for $U / t=4$ and $T=0$. The red dashed line shows the MFT solution [which obeys particle-hole symmetry, $\mu(f)+\mu(1-f)=2 \mu(0.5)]$, but includes regions of unphysical behavior with $\partial \mu / \partial f<0$. The blue line shows the physical solution, which includes regions of phase separation with $\partial \mu / \partial f=0$, accompanied by corresponding gaps at commensurate fillings. Two shaded regions near $f=0.35$ have the same area, according to Maxwell construction.

behavior for this phase around $f=0.2$ (evidenced by the backward curving of the stability region of the checkerboard phase). This is presumably induced by the van Hove singularity, which can stabilize the checkerboard order with the help of thermal activation.

The phase diagram for $T=0$ is shown in Fig. [6] Decreasing the interaction from $U / t=\infty$ causes ordering to disappear for low filling, as the kinetic energy effect is dominant in a dilute system. As the interaction is decreased, the phases with filling near commensurate values are cut off successively. Before its disappearance, phase $5 \mathrm{~B}$ is significantly expanded in filling around $U / t=2.5$, perhaps due to a better nesting of Fermi surface, relative to other phases. Below, but near half filling, decreasing interaction suppresses the $4 \mathrm{D}$ phase in favor of weak order in the 4B phase and eventually leaving only the checkerboard order (2B). The phase separation is not confined to the immediate vicinity of the commensurate fillings, (also seen in Figs. 4 and 5), as it can replace other phases for a range of fillings.

The checkerboard ordering is the most favorable phase at half filling for any value of $U / t$ and it persists down to $U / t=0$, due to perfect nesting of the Fermi surface (see Fig. 7). For large $U / t$, the transition temperature $T_{c}$ is linear in $U$, the largest energy scale. (Since there is only one spin species, there is no energy scale associated with spin exchange, such as $J=4 t^{2} / U$ in the Hubbard model.) For small $U / t, T_{c}$ shows exponentially activated behavior and can be fit with a semi-analytical form, obtained by approximating the non-interacting density of states with a logarithmic divergence representing the van Hove singularity. Similarly, we find an exponentially contracted 

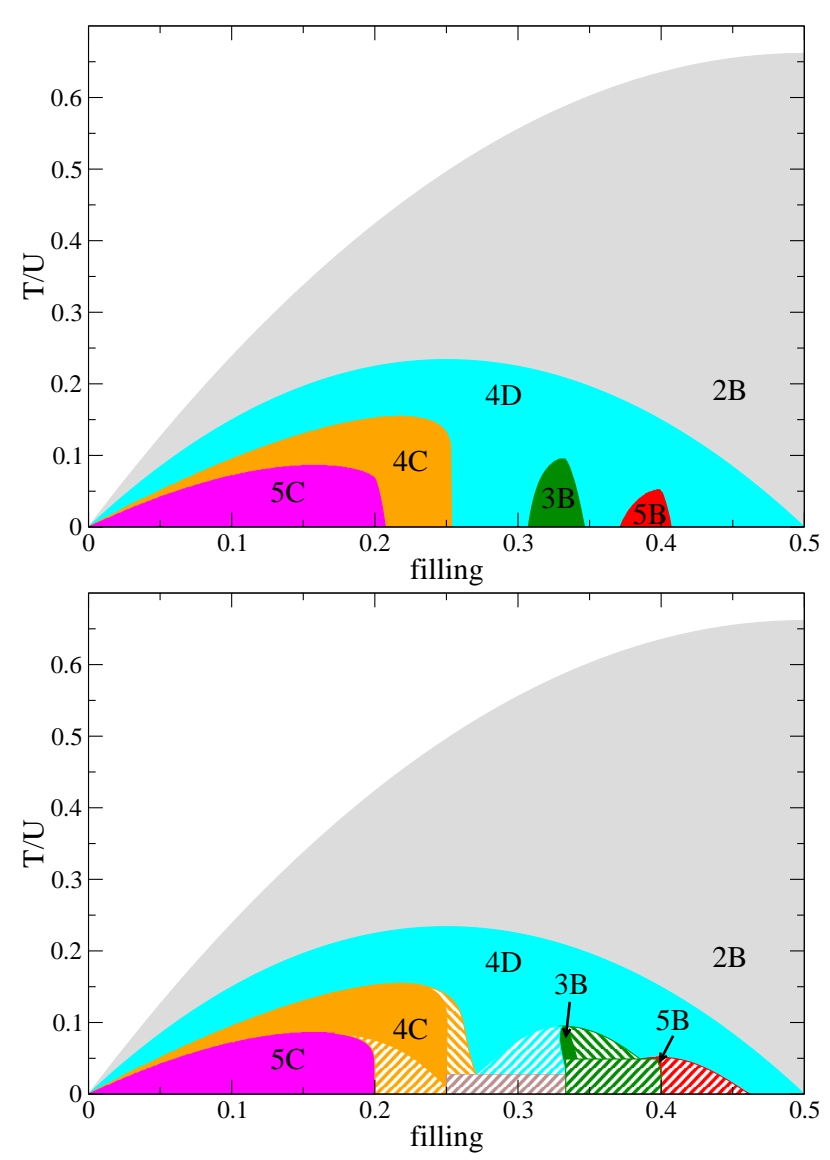

FIG. 4: (color online) $U=\infty$ phase diagram without (top) and with (bottom) phase separation. Phase separation is shown as striped regions. The transitions between $4 \mathrm{D}$ and $2 \mathrm{~B}$ (as well as between $2 \mathrm{~B}$ and $1 \mathrm{~A}$ ) phases are continuous. The rest of the transitions are discontinuous because of phase separation.

range of filling near $U=0$ (see Fig. 6) for this phase. Thus, checkerboard ordering near $U=0$, while possible due to perfect nesting, is strongly suppressed due to the proximity to the van Hove singularity. The gap in the density of states as well as the order parameter at $T=0$ show very similar dependence on $U / t$.

We also report the entropy per particle, since this parameter is important in experiments. Calculated at $T_{c}$ for the checkerboard phase at $f=0.5$, the entropy per particle shows similar behavior with $U / t$ to that of $T_{c}$. However, for $U / t=\infty$, the ordering starts at $S\left(T=T_{c}\right)=2 \ln 2$, clearly an overestimate due to the neglected density fluctuations in the MFT. For smaller $U / t$, a lower entropy state has to be reached to observe the checkerboard order.

The most common experimental test for spatial order is Bragg diffraction of light by the molecules, which directly yields the structure factor in reciprocal space and can be used to identify the order 13. Such an experiment is the analog of an x-ray diffraction experiment on
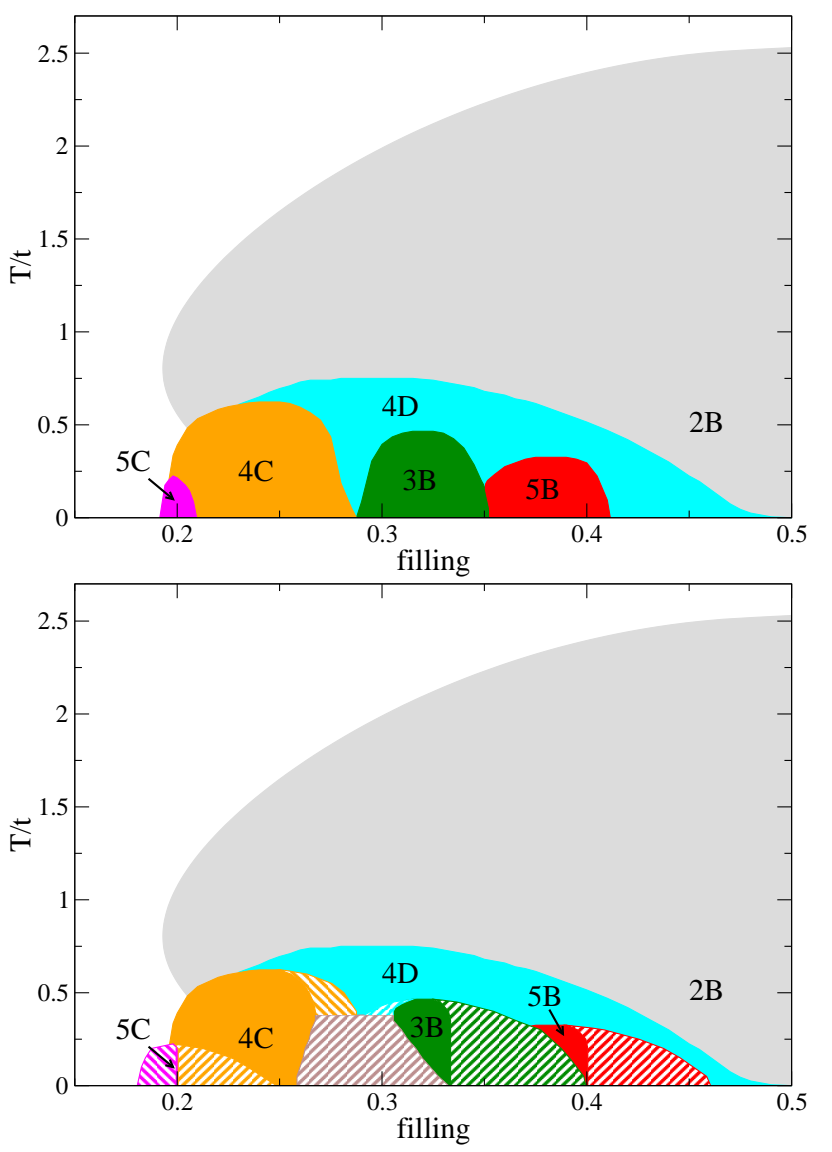

FIG. 5: (color online) $U=4 t$ phase diagram without (top) and with (bottom) phase separation. There is no order at low filling, yet for $f>0.25$, the phase diagram qualitatively resembles the $U / t=\infty$ result. Note the reentrant behavior for the checkerboard phase (2B) around $f=0.2$ and the phase separation between the $5 \mathrm{C}$ and homogeneous phases.

a condensed-matter system used to determine its crystal structure. Table प shows a comparison of different density-wave phases found in this study by listing the real space density patterns, reciprocal space points and structure factors for $U / t=4$. Each of the phases have sufficiently different signatures, both in the location and the relative strength of the peaks of $S(Q)$, making these phases easily identifiable through Bragg diffraction measurements.

Another, less direct way to observe spatial order is time-of-flight measurements that use the expansion of the molecular cloud to infer the initial momentum distribution of the molecules. However, the momentum distribution data do not show prominent differences amongst the different ordered phases, so using this approach to detect the ordering is difficult. 

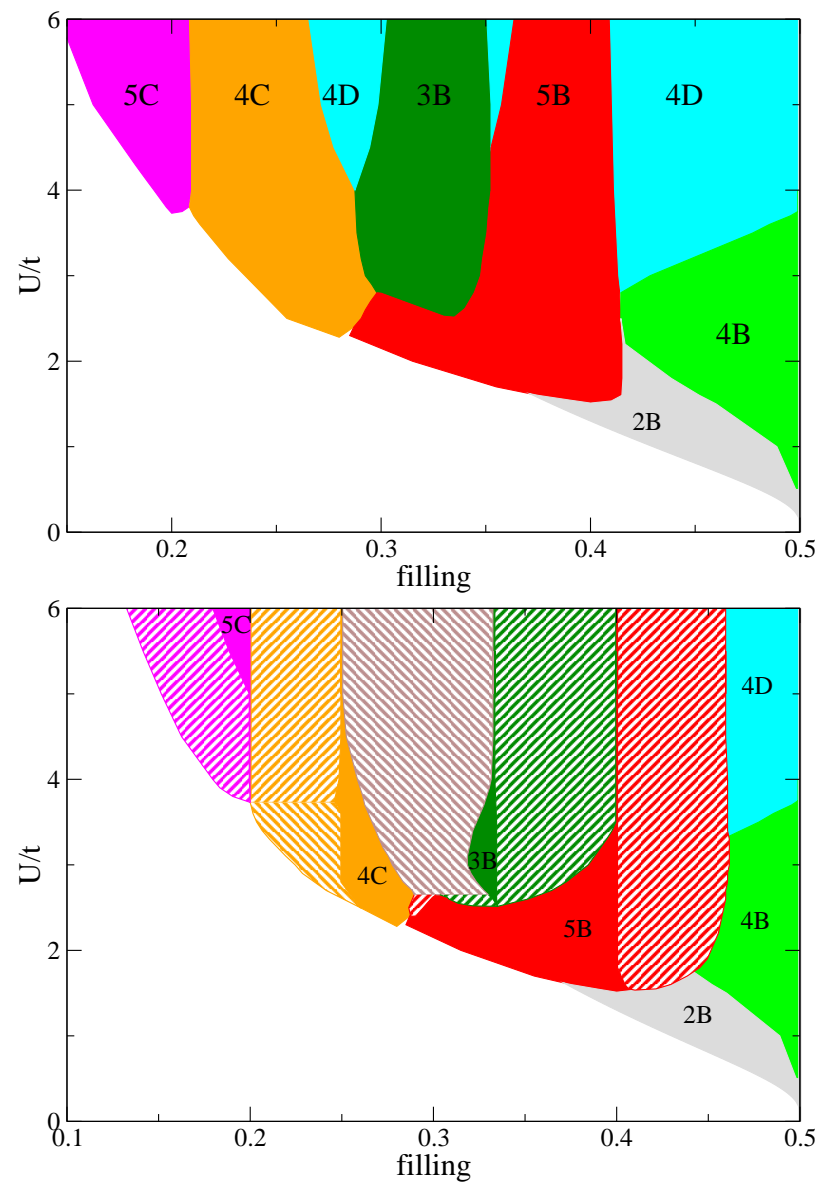

FIG. 6: (color online) $T=0$ phase diagram without (top) and with (bottom) phase separation. Decreasing the interaction contracts the range of filling of the ordered phases and progressively eliminates phases commensurate with low values of filling. Only the checkerboard phase survives down to $U=0$. Phase separation replaces the $4 \mathrm{D}$ phase near $f=0.28$ and $f=0.36$ for larger $U / t$. In parts of the phase diagram, $4 \mathrm{C}$ and $5 \mathrm{C}$ phases show phase separation with the homogeneous state.

\section{DISCUSSION}

The MFT results show a diverse phase diagram with a number of distinct density-wave phases. However, the MFT is an approximate method, which neglects fluctuations and favors ordered states. This brings to mind the question: how many of these density-wave phases are actually present in the exact solution?

In the limit $U / t=\infty$, the Hamiltonian essentially describes a lattice gas model and can be solved numerically without approximation using classical Monte Carlo methods. Preliminary results support the existence of the checkerboard (2B) and 4D phases, although strongly suppressed in temperature and filling, compared to the MFT result. Since the MFT neglects fluctuations, it is not surprising that the range of ordered phases is contracted in

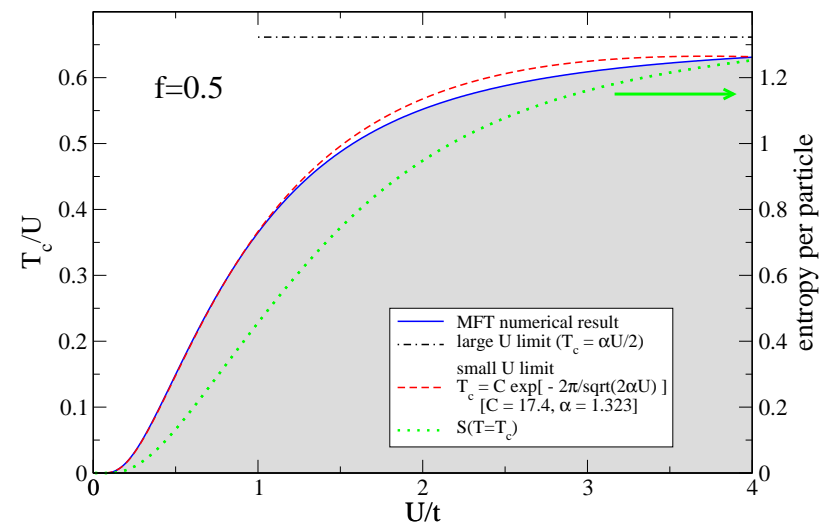

FIG. 7: (color online) $f=0.5$ phase diagram. For large $U / t$, the transition temperature satisfies $T_{c}=\alpha U / 2$, where $\alpha=U[K=(\pi, \pi)]=1.323$. For small $U / t, T_{c}$ fits perfectly to a semianalytical form. The gap in the density of states (not shown) at $f=0.5$ and $T=0$ shows similar dependence on $U / t$. The entropy per particle (dotted line) at $T=T_{c}$ reaches $S=2 \ln 2$ at $U / t=\infty$.

a more precise calculation. However, some higher-period phases become more prominent in the MFT solution for intermediate values of $U / t$, so their presence cannot be entirely ruled out by this $U / t=\infty$ study. A more complete study will be the topic of further research.

Also, the phase separation needs to be reviewed using better approximation techniques. The presence of phase separation is tied to the existence of higher-period phases and the fact that the MFT strongly stabilizes these phases at the corresponding commensurate fillings. Thus, phase separation is promoted by the MFT just like the corresponding ordered phases are. It is possible that including even higher order phases (beyond 4 order parameters) in a MFT study would replace the phaseseparated regions with higher-period ordered phases that are commensurate at the corresponding values of filling, yielding a devil's staircase-like structure. Another possible alternative to phase separation is the formation of a Wigner crystal like state. The reentrant behavior of the checkerboard phase at $U=4 t$ in the MFT results is very likely an artifact of this method and should be reexamined using more precise techniques.

It is notable that the MFT results do not favor stripes that are aligned with the lattice (corresponding to phases $2 \mathrm{~A}, 3 \mathrm{~A}, 4 \mathrm{~A}$ or $5 \mathrm{~A}$ ). We found that these phases are either not stable or they are replaced by other phases with lower free energy. For small $U / t$, good nesting of the Fermi surface appears to be important to lower the energy of the system, while for large $U / t$, strong repulsive interactions between adjacent sites prevents formation of such striped phases. We also see no evidence for any nematic or smectic "liquid-crystal" phases.

Another important question is, which of these densitywave phases can be reached and detected in experiment? The MFT results show large entropy per particle when 


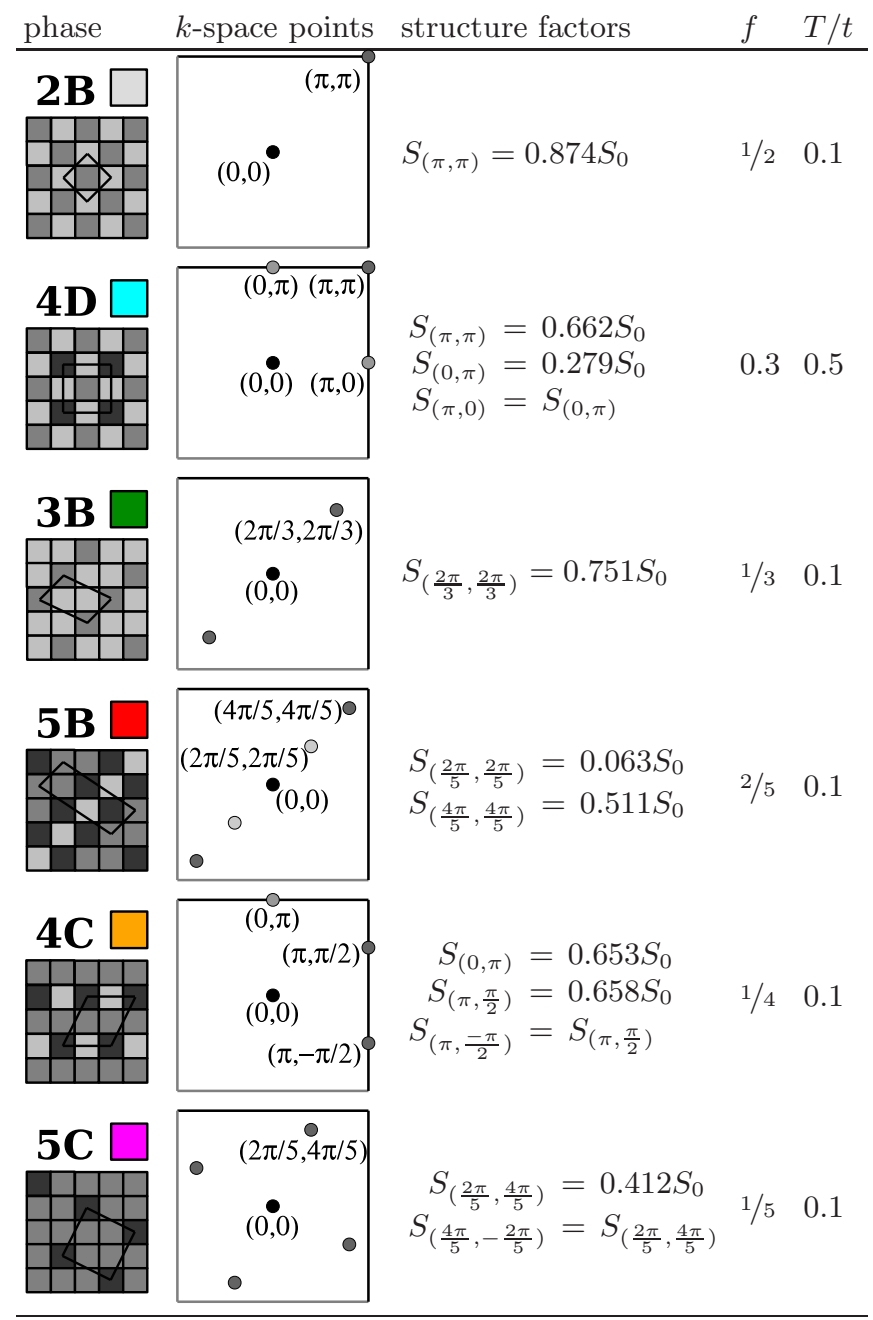

TABLE I: Structure factors for different phases at representative values of filling and temperature for $U / t=4$. The first column shows the real space patterns with darker squares denoting larger average density per site. The second column shows the reciprocal space points corresponding to a given density-wave order, followed by the corresponding structure factors at these points, $S(K)$, relative to $S_{0}=S(K=0)$. Since $S(-K)=S(K)$, only the structure factors for $K_{x} \leq 0$ are shown. The last two columns give the corresponding filling and temperature, respectively.

the checkerboard state develops at half filling for large interaction, but unfortunately the entropy is severely over- estimated in this study, as shown by preliminary MonteCarlo results. The most easily observable features in experiment are the structure factors and the gap in the density of states at the commensurate values of filling. These features are most pronounced for the checkerboard phase. In a trap environment, a large gap at half filling should translate into large spatial regions of checkerboard order if the local density approximation is accurate. Of course, it is not clear if phases that are seen in a homogeneous system are stabilized or destabilized by the trap environment. This is another question to be addressed in a future study.

\section{CONCLUSIONS}

We have studied the ordering of ultracold diatomic gases on a two-dimensional square optical lattice. We have formulated a model, based on spinless fermions with long range dipole interactions, and solved it using mean field theory. We have found a number of possible densitywave orders that occur mostly around the corresponding commensurate values of filling and create gaps in the density of states at these values of the filling. The phase separation is also often seen between different ordered phases in the MFT results. Overall, the checkerboard phase is most stable near half filling and has the highest transition temperature. Due to the lack of an energy scale associated with spin exchange, the transition temperatures increase with interaction strength. Different density wave phases show characteristic and distinct structure factors. We have discussed the validity of these results and the limitations of the MFT method used in this study.

\section{ACKNOWLEDGMENTS}

This work was supported by a MURI grant from Air Force Office of Scientific Research numbered FA9559-091-0617 and from a grant of HPC resources from the Arctic Region Supercomputing Center at the University of Alaska Fairbanks as part of the Department of Defense High Performance Computing Modernization Program. We also acknowedge support from the McDevitt endowment fund.
[1] K. K. Ni, S. Ospelkaus, M. H. G. de Miranda, A. Peer, B. Neyenhuis, J. J. Zirbel, S. Kotochigova, P. S. Julienne, D. S. Jin, and J. Ye, Science 322, 231 (2008).

[2] A. Micheli, G. Pupillo, H. P. Büchler, and P. Zoller, Phys. Rev. A 76, 043604 (2007).

[3] C. Lin, E. Zhao, W. V. Liu, Phys. Rev. B 81, 045115 (2010).

[4] S. Ospelkaus, K.-K. Ni, D. Wang, M. H. G. de Miranda,
B. Neyenhuis, G. Quemener, P. S. Julienne, J. L. Bohn, D. S. Jin, and J. Ye, Science 327, 853 (2010).

[5] A. C. Potter, E. Berg, D.-W. Wang, B. I. Halperin, and E. Demler. Phys. Rev. Lett. 105, 220406 (2010).

[6] G. S. Uhrig, R. Vlaming, Phys. Rev. Lett. 71, 271 (1993).

[7] E. Halvorsen, G. S. Uhrig, G. Czycholl, Z. Phys. B 94, 291 (1994).

[8] G. S. Uhrig, R. Vlaming, Physica B 206, 694 (1995). 
[9] N. G. Zhang and C. L. Henley, Phys. Rev. B 68, 014506 (2003); N.-G. Zhang and C. L. Henley, Eur. Phys. J. B 38, 409 (2004); U. Hizi and C. L. Henley, Europhys. Lett. 65, 228 (2004); S. A. Cheong and C. L. Henley, Phys. Rev. B 74, 165121 (2006).

[10] Y. Yamaguchi, T. Sogo, T. Ito, T. Miyakawa, Phys. Rev. A 82, 013643 (2010).

[11] K. Sun, C. Wu, S. Das Sarma, Phys. Rev. B 82, 075105
(2010).

[12] S. T. Carr, J. Quintanilla, J. J. Betouras, Phys. Rev. B 82, 045110 (2010).

[13] T. A. Corcovilos, S. K. Baur, J. M. Hitchcock, E. J. Mueller, and R. G. Hulet, Phys. Rev. A 81, 013415 (2010). 